\title{
Conducta de forrajeo del gastrópodo Acanthina monodon Pallas, 1774 (Gastropoda: Muricidae) en el intermareal rocoso de Chile central
}

\author{
Foraging behavior of the gastropod Acanthina monodon Pallas, 1774 (Gastropoda: \\ Muricidae) in the intertidal rocky shores of central Chile
}

RUBÉN E. SOTO, JUAN C. CASTILLA \& FRANCISCO BOZINOVIC

\begin{abstract}
Centro de Estudios Avanzados en Ecología \& Biodiversidad, Departamento de Ecología, Facultad de Ciencias Biológicas, Pontificia Universidad Católica de Chile, Casilla 114-D, Santiago, Chile; e-mail: ersoto@bio.puc.cl
\end{abstract}

\begin{abstract}
RESUMEN
En el presente trabajo investigamos aspectos de la ecología y conducta de forrajeo de Acanthina monodon, un gastrópodo murícido que habita en el intermareal rocoso de Chile central. En terreno, estudiamos las variaciones temporales en su distribución, densidad y dieta. En el laboratorio, cuantificamos la tasa de consumo, las preferencias alimentarias, el tiempo de ingestión y la rentabilidad energética obtenida con distintos tipos de presas mediante experimentos y registros en video. Las mayores densidades de individuos de A. monodon fueron observadas en la franja intermareal cercana al nivel cero de marea. En terreno, A. monodon realiza sus actividades de forrajeo principalmente durante la noche y su dieta consistió principalmente de mitílidos (95\%) y cirripedios $(5 \%)$. La composición de la dieta de A. monodon en terreno presentó variaciones temporales las cuales dependerían principalmente de cambios en la oferta de los distintos tipos de mitílidos presentes en terreno durante los dos años de muestreo. En el laboratorio, los individuos de Acanthina presentaron preferencias alimentarias significativas por el mitílido Semimytilus algosus. En general, A. monodon bajo condiciones de laboratorio presentó una conducta de forrajeo en la cual maximizó la ganancia neta de energía, mediante la selección de las especies y tamaños de presas que le retribuyen la mayor rentabilidad energética.
\end{abstract}

Palabras clave: gastrópodo, intermareal, Chile central, forrajeo, selección de presas.

\section{ABSTRACT}

We investigated the ecology and foraging behavior of Acanthina monodon, a muricid gastropod that inhabits in the intertidal rocky shores of central Chile. In the field, we studied temporal variation of their spatial distribution, density, and diet composition. While in the laboratory, we quantified the consumption rate, alimentary preferences, ingestion times and energy profitability obtained with different types of prey using experiments and video recording. High densities of A. monodon individuals were observed in the intertidal fringe near at the level tide zero. In the field, A. monodon actively foraged at night preying mainly on mussels (95\%) and barnacles (5\%). Temporal variation in diet composition of A. monodon was caused mostly by changes in the cover of different mussel species during the two years of sampling. In the laboratory, individual of A. monodon showed significant preference for the mussel Semimytilus algosus. In these experiments, A. monodon's foraging behavior maximized the net gain of energy by selecting species and sizes of prey that provided the greatest energy profitability.

Key words: gastropod, intertidal, central Chile, foraging, prey selection.

\section{INTRODUCCIÓN}

Durante las últimas décadas, el análisis de la conducta asociada a las actividades de alimentación en depredadores marinos ha constituido un foco importante de investigación dentro del ámbito de la ecología (Little \& Kitching 1996, Barnes \& Hughes 1999). Los tipos de depredadores estudiados incluyen a un diverso número de taxa tales como: crustáceos (Elner \& Hughes 1978, Seed \& Hughes 1995), gastrópodos (Hughes 1986, West 1986), equinodermos (Vadas 1977, Lemire \& Himmelman 1996), peces (Kislalioglu \& Gibson 1975, Cáceres et al. 1994) y aves (Sutherland 1982, Kerstein \& Visser 1996). La mayor parte de estas investigaciones han sido realizadas dentro del marco de la teoría de forrajeo y utilizando como base la premisa de maximización de la energía, esto es que la tasa neta de consumo de energía confiere al depredador una ventaja selectiva, la cual se ve 
reflejada en su éxito reproductivo (Schoener 1971, Krebs \& Davies 1997).

La mayoría de los depredadores no consumen las presas en directa proporción con su abundancia, ya que seleccionan determinadas especies y/o tamaños de los individuos (Stephens \& Krebs 1986, Krebs \& Davies 1997). Particularmente, en gastrópodos marinos la selección de dieta puede estar influenciada por un gran número de factores entre los cuales se han señalado: (a) el tamaño del depredador, (b) la detectabilidad de las presas, (c) la accesibilidad de presas (Hughes \& Dunkin 1984, Palmer 1988), (d) la abundancia de presas (Wieters \& Navarrete 1998) y (e) variaciones en la distribución espacial de los depredadores (Hughes 1986, Rochette \& Dill 2000).

Los estudios realizados acerca de la conducta de forrajeo de los gastrópodos depredadores que habitan en el intermareal rocoso muestran que ellos son potencialmente capaces de discriminar distintos tipos de presas de acuerdo a su calidad energética (Kohn 1983, Hughes 1986). Las diferencias en la calidad de presas están dadas principalmente por su tamaño, componentes nutricionales y tiempo de manipulación (Carriker 1981, Palmer 1984, 1988). Una vez que el gastrópodo ha realizado la categorización de las presas, dentro de la teoría de forrajeo, específicamente el modelo de selección de presas, predice que aquellas de mayor calidad energética serán seleccionadas en primer lugar, mientras que la ingestión de las presas energéticamente subóptimas dependerá de la disponibilidad de presas óptimas en el ambiente (Fairweather 1985, Burrows \& Hughes 1991). De la misma manera, los modelos de selección de presas predicen que un consumidor debe seleccionar su dieta basándose en la rentabilidad de los tipos de presas disponibles. La rentabilidad es generalmente expresada como el contenido energético de la presa dividido por las unidades de tiempo utilizado por el depredador en la búsqueda, manipulación y consumo de un determinado tipo de ellas (Hughes 1986, 1988, Stephens \& Krebs 1986).

En la costa de Chile existe una gran diversidad de especies de gastrópodos marinos que actúan como depredadores (Vermeij 1978, Castilla 1981, Castilla \& Paine 1987). Sin embargo, existe escasa información respecto a sus conductas de forrajeo. La especie más estudiada en cuanto a su conducta de alimentación ha sido el murícido Concholepas concholepas (Castilla et al. 1979, Méndez \& Cancino 1990, Navarro \& Torrijos 1995, Serra et al. 1997) debido principalmente a su importancia económica (Castilla 1999) y al significativo efecto que produce esta especie sobre la estructuración y composición de las comunidades rocosas intermareales (Durán \& Castilla 1989, Castilla 1999).

El murícido Acanthina monodon es un gastrópodo carnívoro que depreda activamente sobre mitílidos y cirripedios y habita en plataformas rocosas de la zona intermareal en la costa centro y sur de Chile (Poblete et al. 1987). Estudios preliminares acerca de su conducta de forrajeo han sido realizados en la zona de Valdivia (sur de Chile), aunque todas las investigaciones han sido orientadas hacia aspectos comunitarios, específicamente a efectos subletales sobre sus potenciales presas (López et al. 1995, Moreno 1995), o competencia por recursos alimentarios con $C$. concholepas (Dye 1991). Sin embargo, para las poblaciones que habitan en Chile central se desconocen aspectos básicos relacionados con su distribución espacial, abundancia o dieta.

Dado que la puesta a prueba de hipótesis en teoría de forrajeo contemporánea requiere de antecedentes básicos de la conducta de los consumidores y de aspectos ecológicos de las presas y tomando en cuenta el contexto teórico y los antecedentes anteriormente señalados, los objetivos del presente trabajo fueron: (1) determinar la conducta de forrajeo de $A$. monodon, caracterizando las preferencias alimentarias y el tiempo de manipulación en relación con distintos tipos de presas; (2) Cuantificar la abundancia, distribución espacial y la dieta de esta especie y su relación con la variabilidad temporal en la oferta de presas en plataformas intermareales rocosas de Chile central. Con lo anterior se examina y somete a prueba la hipótesis general de este estudio, la cual indica que A. monodon se comporta como un maximizador de energía durante las actividades relacionadas con el forrajeo. De este modo, A. monodon selecciona las especies y tamaños de presas que le entreguen la mayor rentabilidad energética.

\section{MATERIALES Y MÉTODOS}

\section{Área de estudio}

Los muestreos y registros en terreno fueron realizados en plataformas rocosas intermareales ubicadas aproximadamente a dos kilómetros al norte de la localidad de Las Cruces en Chile central $\left(33^{\circ} 38^{\prime} 44,9^{\prime}\right.$ ' S, $71^{\circ} 28^{\prime} 51,9^{\prime}$ 'O). La composición y estructura comunitaria del ambiente intermareal rocoso 
de Chile central han sido descritas por diversos autores (i.e., Castilla 1981, Castilla \& Paine 1987, Santelices 1991).

\section{Densidad y distribución espacial}

Para determinar la distribución espacial de los individuos de $A$. monodon dentro de plataformas rocosas intermareales, se realizaron transectos perpendiculares a la línea de costa en cinco plataformas elegidas al azar dentro del área de muestreo. En cada una de las plataformas se midió la densidad de $A$. monodon dentro de cuadrantes de $1,0 \times 0,5 \mathrm{~m}$. Los cuadrantes fueron localizados cada $0,5 \mathrm{~m}$ iniciando el muestreo desde el nivel ubicado un metro bajo de la cota cero de altura de marea (Servicio Hidrográfico y Oceanográfico de la Armada de Chile 1999), posteriormente el muestreo fue repetido posicionando el cuadrante en el siguiente segmento de la plataforma hasta llegar a los dos metros sobre el nivel cero de marea (Castilla 1988, Rochette \& Dill 2000). Este muestreo fue repetido en cuatro oportunidades durante el año 1999, que se corresponden con evaluaciones en distintas estaciones del año de manera de caracterizar la variabilidad temporal del patrón. Los datos fueron analizados mediante un análisis de la varianza de tipo factorial (Zar 1996) usando la densidad como variable de respuesta; la altura de marea y estación del año fueron utilizados como factores. El análisis fue realizado una vez que los datos fueron transformados mediante la aplicación de logaritmo del valor +1 , para satisfacer los supuestos de normalidad y de homogeneidad de las varianzas necesarios para el análisis (Underwood 1997).

\section{Ritmo de alimentación}

Con la finalidad de caracterizar en terreno la ventana temporal en la cual $A$. monodon efectúa el consumo de presas y su relación con el ciclo mareal y diario, se realizaron muestreos con una metodología similar a la descrita para el análisis de la dieta. Este muestreo fue repetido en cuatro ocasiones, bajo distintos escenarios ambientales: (a) marea baja diurna, (b) marea alta diurna, (c) marea baja nocturna (d) y marea alta nocturna. En cada una de estas oportunidades se registró si los individuos estaban consumiendo presas o se encontraban inactivos refugiados en grietas, los muestreos se realizaron hasta completar un total de 100 individuos observados en cada oportunidad. Los muestreos efectuados durante la noche fueron realizados utilizando linternas. Mientras que evaluaciones efectuadas durante marea alta fueron realizadas durante períodos de mar llana usando equipo de buceo apnea. Los datos fueron analizados mediante el uso de tablas de contingencia utilizando como estadístico la prueba de probabilidad exacta de Fisher (Zar 1996).

\section{Dieta}

Para identificar cuáles eran las especies de invertebrados consumidas por A. monodon en terreno, se realizaron transectos paralelos a la línea de costa durante períodos de marea baja nocturna en busca de individuos que estuviesen alimentándose. Una vez que un individuo era localizado se consideró que estaba consumiendo una presa si se encontraba sobre ella perforando o ingiriéndola, posteriormente se identificó la presa consumida, dentro de lo posible a nivel de especie. El muestreo fue realizado hasta evaluar al menos 50 individuos de A. monodon que estuvieran alimentándose. Este tipo de registro fue repetido en ocho oportunidades en los años 1999 y 2000 para determinar posibles variaciones temporales en la conducta de alimentación. Para comparar la frecuencia de consumo de mitílidos y cirripedios durante las cuatro estaciones de los años 1999 y 2000 se utilizó un análisis de varianza (ANDEVA) de una vía previa transformación de los datos (porcentajes) mediante el arco seno de la raíz cuadrada de la proporción para satisfacer los supuestos del ANDEVA (Underwood 1997). Las frecuencias relativas de datos de consumo de los distintos tipos de presas fueron analizados mediante el uso de tablas de contingencia de dos vías (Zar 1996).

\section{Relación longitud peso}

Para caracterizar la relación entre la longitud de la concha y el peso húmedo de los individuos de A. monodon, se midieron y pesaron 160 individuos de tamaños corporales que fluctuaron desde 7 a $45 \mathrm{~mm}$ aproximadamente. Las medidas morfométricas de las conchas fueron evaluadas siguiendo los criterios propuestos por Poblete et al. (1987), utilizando para ello un pie de metro digital con una precisión de $0,1 \mathrm{~mm}$. El peso húmedo de los individuos fue evaluado utilizando una balanza analítica $( \pm 0,001 \mathrm{~g})$, previa limpieza de las conchas, para eliminar epibiontes. Con los valores obtenidos se ajustó una relación entre la longitud de la concha $(\mathrm{mm})$ y el peso corporal (g) utilizando una ecuación exponencial. Posteriormente, mediante la aplicación de logaritmo a los datos se determinó el 
coeficiente de regresión y la probabilidad asociada a la curva obtenida mediante el uso de un ANDEVA de una vía (Zar 1996).

\section{Oferta de presas en terreno}

Para cuantificar la oferta de presas en terreno se realizaron mediciones de la cobertura de organismos sésiles utilizando un cuadrante de 50 x $50 \mathrm{~cm}$ aplicando el método intersección de puntos (Foster et al. 1991). Este muestreo fue realizado mensualmente desde enero de 1999 hasta diciembre de 2000. En cada una de las oportunidades, se midió la cobertura de presas en 10 cuadrantes seleccionados al azar en la zona inferior del intermareal de a lo menos cinco plataformas rocosas ubicadas en el sitio de muestreo descrito anteriormente. Los datos obtenidos fueron comparados mediante un análisis de varianza de medias repetidas, en que se utilizaron las distintas especies de presas como variable categórica y los meses como variable temporal (Zar 1996). El análisis fue realizado previa transformación de los datos (porcentajes) mediante el arco seno de la raíz cuadrada de la proporción para satisfacer los supuestos del ANDEVA (Underwood 1997).

\section{Relación longitud peso de las presas}

Para evaluar de qué manera se relacionaban la longitud de la concha y el peso seco de las partes blandas de las presas, se disecaron 80 individuos de Semimytilus algosus y $80 \mathrm{de}$ Perumytilus purpuratus de entre 3 y $40 \mathrm{~mm}$ de longitud máxima de concha. La recolección de individuos desde terreno fue realizada durante julio de 1999. Para cada uno de los individuos se midió la longitud, altura y ancho máximos de la concha con un pie de metro digital de 0,1 $\mathrm{mm}$ de precisión. En los individuos disecados se separaron las valvas y los tejidos blandos, excluyendo el biso, los que posteriormente fueron mantenidos en una estufa a $70{ }^{\circ} \mathrm{C}$ por 24 $\mathrm{h}$, hasta lograr su peso constante. Esto permitió registrar para cada mitílido el peso seco tanto de los tejidos blandos como de las valvas mediante una balanza analítica $( \pm 0,001 \mathrm{~g})$.

Por otra parte, con los tejidos blandos secos de individuos adultos se determinó el contenido energético de ellos utilizando una bomba calorimétrica (PAR 12). Se realizaron determinaciones en cinco réplicas y se consideró el valor, libre de ceniza, cuando las diferencias entre réplicas fueron menores al $1 \%$.

Con los valores obtenidos se ajustó una relación entre la longitud de la valva $(\mathrm{mm})$ y el peso seco de la carne (g) utilizando una ecuación exponencial. Posteriormente, mediante la aplicación de logaritmo a los datos se determinó el coeficiente de regresión y la probabilidad asociada a la curva obtenida mediante el uso de un ANDEVA de una vía (Zar 1996).

Determinación del grosor de la concha de las presas

Para cuantificar el grosor de la concha se utilizó el índice propuesto por Guiñez (1996), quien lo considera como la razón entre el peso seco de las valvas dividido por el área superficial de la concha, multiplicado por 1.000. Por medio de este índice se realizaron comparaciones entre el grosor de la concha de las especies de mitílidos consumidas por el gastrópodo A. monodon. A los valores obtenidos se les ajustó una relación entre la longitud $(\mathrm{mm})$ y grosor de la concha utilizando una ecuación de tipo potencial. Posteriormente, se realizó una comparación entre las relaciones del grosor y la longitud de la concha para las dos especies de mitílidos evaluados mediante el uso de un análisis de la covarianza (ANCOVA, Zar 1996).

\section{Experimentos en laboratorio}

Los individuos de A. monodon y las presas que fueron utilizados en los experimentos y registros en el laboratorio fueron recolectados manualmente desde plataformas intermareales ubicadas en el sitio de muestreo. Los caracoles recolectados fueron trasladados a los laboratorios de la Estación Costera de Investigaciones Marinas (ECIM) en Las Cruces, donde fueron mantenidos en acuarios con agua de mar circulante y aireación constante. La temperatura del agua de mar utilizada en todos los experimentos y evaluaciones en el laboratorio fluctuó entre los 12 y $14{ }^{\circ} \mathrm{C}$.

Las presas fueron recolectadas poniendo especial cuidado en que todos los individuos fueran de una misma área del intermareal para estandarizar variaciones en las conchas y contenido de carne que pudiesen estar asociadas con la exposición al oleaje, elevación intermareal o acción de depredadores (Guiñez 1996). Las presas obtenidas fueron llevadas al laboratorio donde fueron mantenidas en cajas de plástico perforadas de 22 x $15 \times 7 \mathrm{~cm}$, sumergidas en agua de mar circulante y aireación constante. Todos los experimentos fueron realizados dentro de bandejas de fibra de vidrio de $200 \times 80 \times 15 \mathrm{~cm}$, con agua de mar 
circulante en las cuales, mediante la utilización de un programador horario para riego de jardines marca Nelson (modelo 5800E), se simuló la variación de mareas. La simulación de subida y bajada de marea $(15 \mathrm{~cm}$ de altura) fue implementada cada seis horas con un adelantamiento de media hora cada día de acuerdo a los registros de las tablas de marea correspondientes al puerto de San Antonio (Servicio Hidrográfico y Oceanográfico de la Armada de Chile 1999).

\section{Tasa de consumo}

Para cuantificar la tasa de consumo de presas de los individuos de A. monodon, se seleccionaron 40 individuos de entre 12 y 45 $\mathrm{mm}$ de longitud, 20 de ellos fueron alimentados con $S$. algosus y 20 con $P$. purpuratus. Los caracoles fueron instalados individualmente en cajas de plástico perforadas de un litro de capacidad $(22 \times 15 \times 7 \mathrm{~cm})$. A cada individuo se le ofrecieron simultáneamente diez presas de distintos tamaños corporales que abarcaban desde los 5 a los $30 \mathrm{~mm}$ de longitud de concha. Las cajas experimentales fueron sumergidas parcialmente en bandejas de madera recubiertas con fibra de vidrio en las cuales se mantuvieron con circulación de agua de mar y aireación constante. Diariamente se revisaron y midieron mediante un pie de metro las presas que habían sido consumidas siendo reemplazadas por una semejante tanto en especie como tamaño corporal. El registro de la actividad de forrajeo se realizó durante julio de 1999 y fue mantenido por tres semanas. A los valores obtenidos se les ajustó una relación entre el tamaño corporal de A. monodon y la tasa de consumo por medio de una ecuación de tipo potencial para ambos tipos de presas evaluadas. Posteriormente, se realizó una comparación entre la relación del tamaño corporal del depredador (g) y su respectiva tasa de consumo para las dos especies de mitílidos utilizando un análisis de la covarianza (Zar 1996).

\section{Preferencias alimentarias}

Para cuantificar las preferencias alimentarias de los individuos de A. monodon se realizaron experimentos de selección múltiple de presas. Se seleccionaron 15 caracoles adultos y 15 juveniles los cuales fueron instalados individualmente en cajas de plástico perforadas de un litro de capacidad. A cada uno de los individuos se les ofreció simultáneamente tres tipos de presas de un tamaño corporal semejante $(20 \mathrm{~mm})$ : los mitílidos $P$. purpuratus, S. algosus y Brachidontes granulata. Del mismo modo, para estimar la mortalidad natural de las presas durante la realización del experimento, se implementaron tratamientos controles, consistentes en cajas sin A. monodon para cada uno de los tipos de presas evaluadas. Al inicio del experimento las presas fueron distribuidas homogéneamente sobre el fondo de las cajas. El consumo de alimento fue controlado diariamente y las presas consumidas fueron identificadas $y$ medidas utilizando un pie de metro digital. Durante cada control se renovaron las presas consumidas por una de tipo semejante para mantener la oferta constante, además las presas se redistribuyeron homogéneamente en el fondo de cada caja. El experimento se mantuvo durante ocho días y fue implementado en un sistema de bandejas con agua de mar circulante, aireación constante mediante piedras de burbujeo y régimen de simulación de marea.

Utilizando un diseño experimental semejante al descrito en el párrafo anterior también se cuantificaron las preferencias alimentarias para 15 individuos juveniles de $A$. monodon. Los datos de consumo tanto de los individuos adultos como juveniles fueron analizados utilizando la prueba no paramétrica de Friedman (Connover 1981, Roa 1992).

\section{Preferencias alimentarias por tamaños de presa}

Para caracterizar qué tamaños corporales de presas eran preferentemente seleccionados por los individuos adultos de A. monodon se realizaron experimentos de selección múltiple en el laboratorio. Se usaron 30 caracoles de tamaños corporales entre 33 a $35 \mathrm{~mm}, 15$ de ellos fueron sometidos a experimentos de selección con el mitílido $P$. purpuratus y 15 con $S$. algosus. Cada uno de los caracoles fue instalado individualmente en cajas de plástico $(12 \times 12 \times 7 \mathrm{~cm})$ en donde se le ofrecieron nueve presas de mitílidos de tres tamaños distintos: pequeños (15 a $20 \mathrm{~mm}$ ), medianos $(20,1$ a $25 \mathrm{~mm})$ y grandes $(25,1$ a $30 \mathrm{~mm})$. El consumo de alimento fue controlado diariamente y las presas consumidas fueron medidas utilizando un pie de metro digital. Durante cada control se renovaron las presas consumidas por una de tamaño corporal semejante para mantener la oferta constante; además, se redistribuyeron las presas homogéneamente en el fondo de cada caja. El experimento fue mantenido durante 8 días y se implementó en un sistema de bandejas con agua de mar circulante y aireación constante mediante piedras de burbujeo. Los datos de 
consumo tanto de los individuos que estaban consumiendo Perumytilus como Semimytilus fueron analizados mediante la prueba no paramétrica de Friedman (Conover 1981, Roa 1994).

\section{Tiempo de manipulación e ingestión de presas}

Para cuantificar el tiempo requerido por los individuos adultos de A. monodon durante la manipulación e ingestión de presas de distintos tamaños corporales, se realizaron registros mediante una cámara de video (Sony, modelo Handycam). Los registros de tiempo de manipulación fueron realizados utilizando como presas los mitílidos $P$. purpuratus y $S$. algosus de tamaños corporales que fluctuaban entre 10 y $30 \mathrm{~mm}$ de longitud de concha. Las cintas fueron revisadas mediante un televisor registrándose el tiempo ( $\pm 1 \mathrm{seg}$ ) ocupado por cada unos de los caracoles en la ingestión y manipulación de un determinado tamaño de presa de mitílido. Para los cálculos de la rentabilidad energética $\left(R_{x}\right)$ de cada una de las especies y tamaños de presas evaluadas se utilizó la razón entre el tiempo requerido para el consumo (t), (manipulación más ingesta) y el aporte energético (E) de la presa consumida $\left(R_{x}=E / t\right.$, Elner \& Hughes 1978). Los datos de tiempo de manipulación fueron analizados mediante el ajuste de una relación entre el tamaño corporal de la presa y el tiempo de consumo por medio de una ecuación de tipo potencial para ambos tipos de presas evaluadas. Mediante la aplicación de logaritmo a los datos se determinó el coeficiente de regresión y la probabilidad asociada para cada una de las curvas obtenidas mediante el uso de un ANDEVA de una vía (Zar 1996).

\section{RESULTADOS}

\section{Densidad y distribución espacial}

La distribución espacial de los individuos de $A$. monodon estuvo circunscrita a una franja específica dentro de la zona intermareal rocosa de Chile central. Esta franja alcanza un ancho aproximado de dos metros y se ubicó un metro tanto bajo como sobre el nivel cero de altura de marea en la zona intermareal (Fig. 1). En general, las mayores densidades de A. monodon fueron observadas en la franja intermareal cercana al nivel cero de marea, lo cual fue independiente de la estación del año. El análisis de varianza indicó que la altura de marea constituye un factor significativo para explicar las variaciones en la distribución espacial de los individuos de A. monodon, mientras que no se observaron variaciones estadísticamente significativas en la distribución espacial en relación con los cambios estacionales (Tabla 1).

\section{Ritmo de alimentación}

Los individuos de A. monodon realizan sus excursiones $y$ actividades de forrajeo principalmente en la noche, ya que durante este período cerca del $80 \%$ de los individuos se encontró en fase de alimentación, mientras que en los muestreos realizados durante el día un número cercano al $10 \%$ estaban en dicha fase (Fig. 2). Al analizar cuantitativamente la actividad de forrajeo y su asociación con el ciclo diario y mareal fue posible determinar que A. monodon consume sus presas durante la noche (prueba de probabilidad exacta de Fisher, $\mathrm{P}<0,001)$, lo que fue independiente del nivel de marea en que se realizó el muestreo (prueba de Fisher, $\mathrm{P}=0$,514, Fig. 2).

TABLA 1

Análisis de varianza para la distribución espacial de Acanthina monodon y su relación con la estación del año y la altura de marea

Analysis of variance for spatial distribution of Acanthina monodon and their relationship with season and tide height

\begin{tabular}{lccccc}
\hline $\begin{array}{l}\text { Fuente de } \\
\text { variación }\end{array}$ & $\begin{array}{c}\text { Suma de } \\
\text { cuadrados }\end{array}$ & $\begin{array}{c}\text { Grados de } \\
\text { libertad }\end{array}$ & $\begin{array}{c}\text { Cuadrado } \\
\text { medio }\end{array}$ & Valor de F & Valor de P \\
\hline Estación & 0,704 & 3 & 0,234 & 0,344 & 0,793 \\
Altura de marea & 41,688 & 3 & 13,896 & 20,399 & 0,000 \\
Estación x altura marea & 6,107 & 9 & 0,678 & 0,996 & 0,453 \\
Error & 0,679 & 64 & 0,681 & & \\
\hline
\end{tabular}



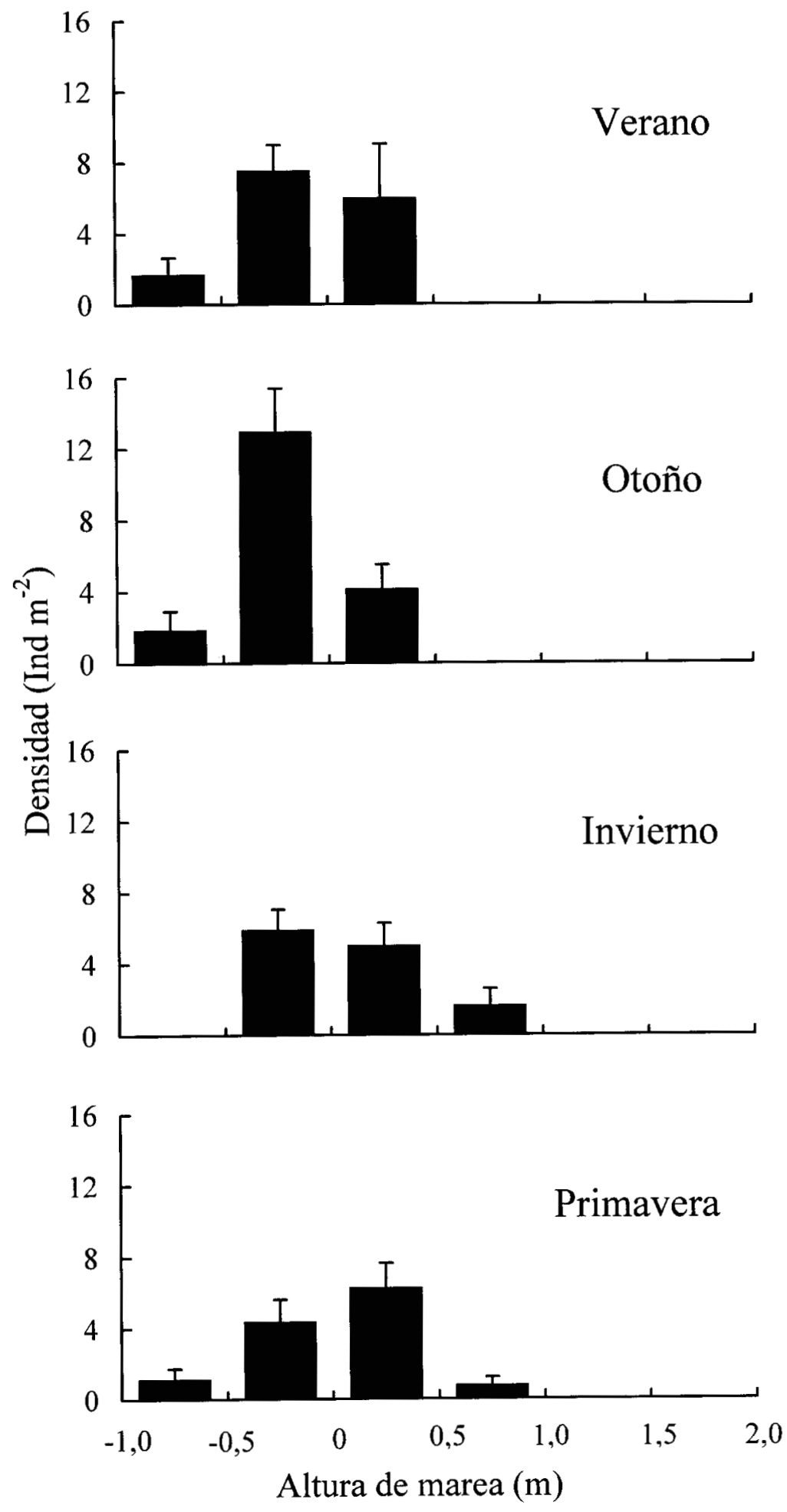

Fig. 1: Densidad promedio y distribución espacial del gastrópodo Acanthina monodon durante cuatro estaciones del año 1999 en el intermareal rocoso de Chile central. Las líneas sobre las barras indican 1 error estándar.

Mean density and spatial distribution of gastropod Acanthina monodon during four seasons in 1999 on intertidal rocky shores of central Chile. Lines above bars indicate 1 standard error. 


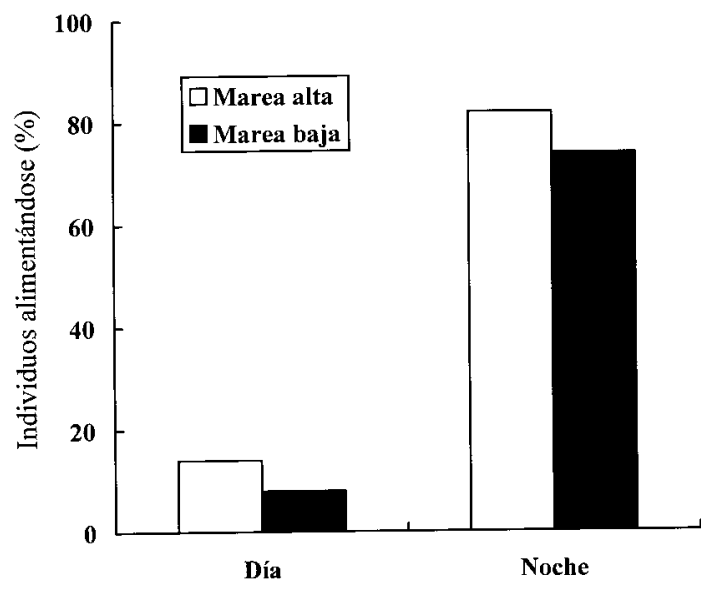

Fig. 2: Porcentaje de individuos de Acanthina monodon alimentándose en terreno durante el día y la noche en condiciones de marea alta y baja.

Percentage of individuals of Acanthina monodon in the field feeding during day and night time under high and low tide conditions.

\section{Dieta}

La dieta de A. monodon en el intermareal rocoso de Chile central consistió principalmente de dos tipos de invertebrados: mitílidos y cirripedios. Entre los mitílidos consumidos se encontraban $S$. algosus, $P$. purpuratus y $B$. granulata, mientras que entre los cirripedios se incluían Balanus laevis, Balanus flosculus, Nothochtamalus scabrosus, Jehlius cirratus e individuos juveniles de Austromegabalanus psittacus. Al cuantificar los tipos de presas consumidas por A. monodon en terreno se constató que los mitílidos constituyen cerca del $95 \%$ de su dieta (Fig. 3). Las comparaciones estadísticas del consumo de presas indicaron que tanto para el año 1999 (ANDEVA, $\left.\mathrm{F}_{1,6}=728,72 ; \mathrm{P}<0,0001\right)$, como para el año 2000 (ANDEVA, $\mathrm{F}_{1,6}=953,60 ; \mathrm{P}<$ $0,0001)$ existió un consumo significativamente mayor de presas pertenecientes al grupo de los mitílidos que al de los cirripedios.

La frecuencia estacional de consumo de presas en terreno para los años 1999 y 2000 presentó diferencias interanuales significativas (Chi-cuadrado $=52,16 ; \mathrm{gl}=2 ; \mathrm{P}<0,0001$; Fig. 3). Estas diferencias estuvieron principalmente asociadas a que durante el año 1999 se presentaron variaciones intraanuales $($ Chi-cuadrado $=64,72 ; \mathrm{gl}=6 ; \mathrm{P}<0,001)$ dadas por estaciones en que la mayor frecuencia de consumo por parte de Acanthina fue sobre $S$. algosus (verano y otoño), mientras que durante las estaciones de invierno y primavera la mayor frecuencia de consumo fue sobre $P$. purpuratus (Fig. 3). En los muestreos del año 2000, P. purpuratus constituyó la presa con mayor frecuencia de consumo no observándose diferencias significativas durante las cuatro estaciones evaluadas (Chi-cuadrado $=3,52 ; \mathrm{gl}=6 ; \mathrm{P}=$ $0,7402)$.

\section{Relación longitud peso en A. monodon}

La relación entre la longitud de la concha y el peso corporal para los individuos de $A$. monodon recolectados en el intermareal rocoso de Las Cruces se muestra en la Fig. 4.

\section{Oferta de presas en terreno}

Se observó una gran variación temporal en la oferta ambiental de las presas potenciales disponibles para el consumo del gastrópodo $A$. monodon. Durante los muestreos realizados en el año 1999 se observó que en los meses de verano existió una mayor oferta relativa de $S$. algosus, mientras que a partir de julio la presa predominante fue $P$. purpuratus (Fig. 5). Por su parte, en el año 2000 la presa con mayor cobertura (oferta) fue el mitílido P. purpuratus. Una constante durante los dos años de muestreo estuvo dada por la baja cobertura de cirripedios, con valores que no sobrepasaron el $10 \%$ (Fig. 5). Un análisis de varianza de medias repetidas mostró que existían diferencias significativas entre la cobertura de los distintos tipos de especies que pueden constituir potenciales presas de A. monodon $\left(\mathrm{F}_{2,12}=135,09 ; \mathrm{P}<0,0001\right)$. Por otra parte, existe una interacción significativa entre el tiempo de muestreo y la cobertura de los tipos de presas $\left(\mathrm{F}_{2,40}=5,9 ; \mathrm{P}<0,001\right)$.

\section{Relación longitud peso de las presas}

Las curvas que relacionan el tamaño corporal (longitud de la concha) y peso seco de los tejidos blandos para los mitílidos $S$. algosus y $P$. purpuratus, se muestran en la Fig. 6. Un análisis de la covarianza mostró diferencias significativas en la relación, entre ambas especies de mitílidos, dado que el peso seco de las partes blandas presentaba valores diferentes para un mismo tamaño corporal de mitílido $\left(\mathrm{F}_{1,151}=61,48 ; \mathrm{P}<0,0001\right)$. El contenido energético promedio de $P$. purpuratus fue de $21,9 \mathrm{~kJ} \mathrm{~g}^{-1}(\mathrm{DE}=0,53)$ mientras que para $S$. algosus se registró un valor promedio de $22,2 \mathrm{~kJ} \mathrm{~g}^{-1}(\mathrm{DE}=0,09)$ libre de ceniza. 
AÑO 1999
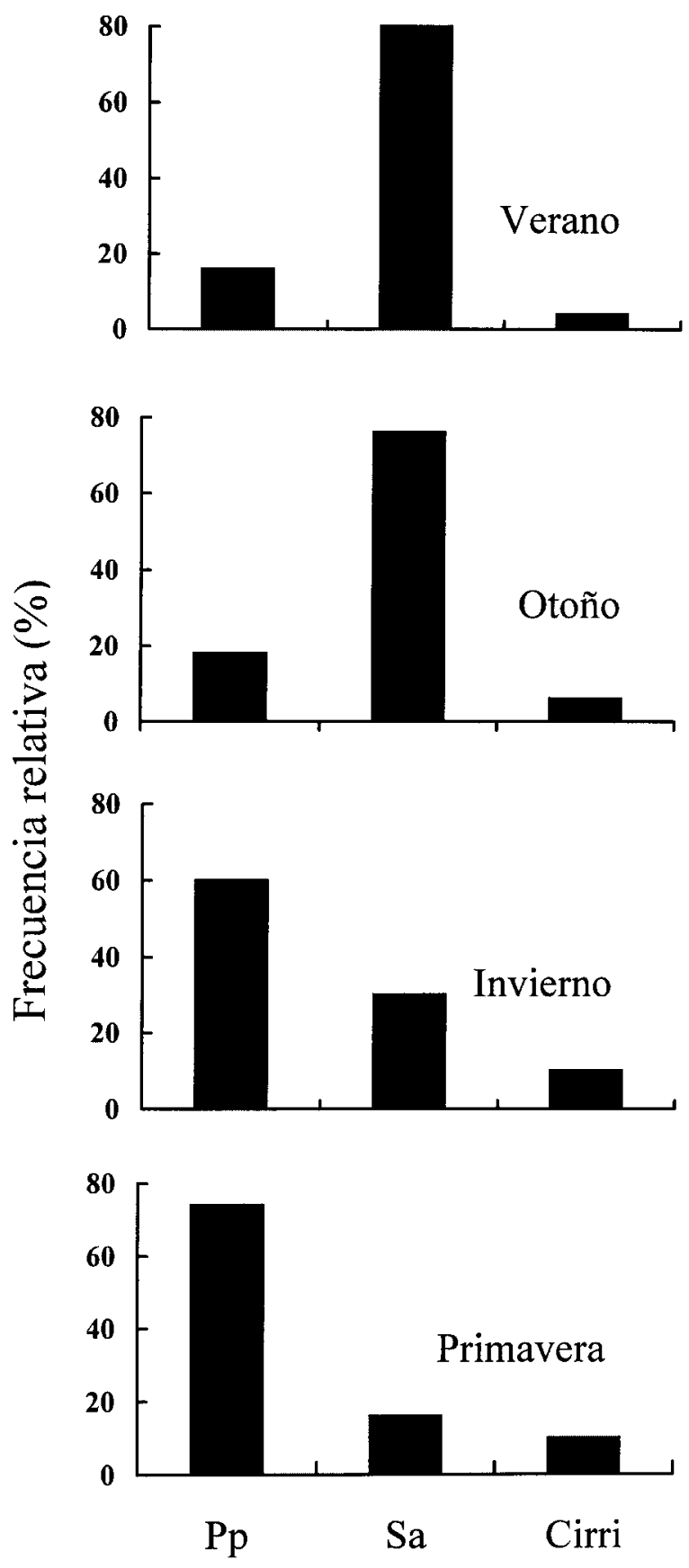

AÑO 2000
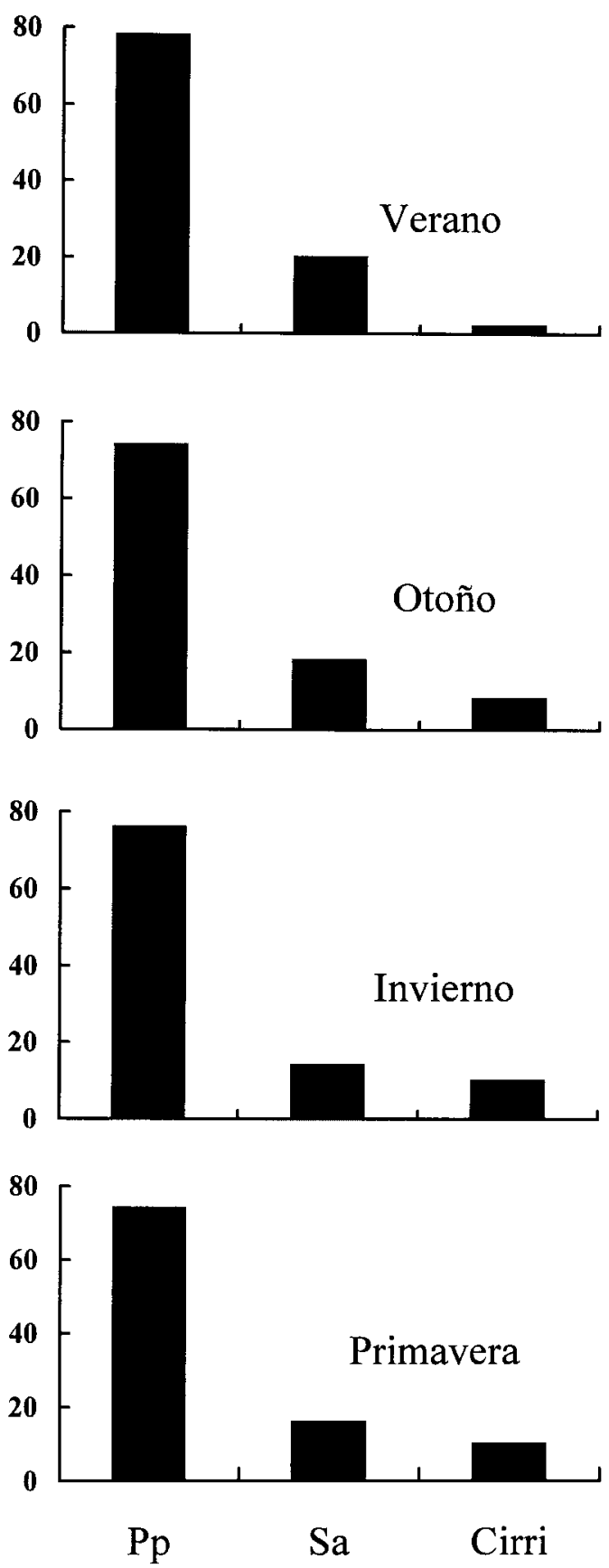

Fig. 3: Frecuencia relativa de la composición dietaria de Acanthina monodon evaluada en terreno durante los años 1999 y 2000; $\mathrm{Pp}=$ Perumytilus purpuratus, $\mathrm{Sa}=$ Semimytilus algosus y Cirri $=$ cirripedios.

Relative frequency of diet composition of Acanthina monodon evaluated in the field during 1999 and 2000; Pp = Perumytilus purpuratus, $\mathrm{Sa}=$ Semimytilus algosus and Cirri $=$ barnacles. 


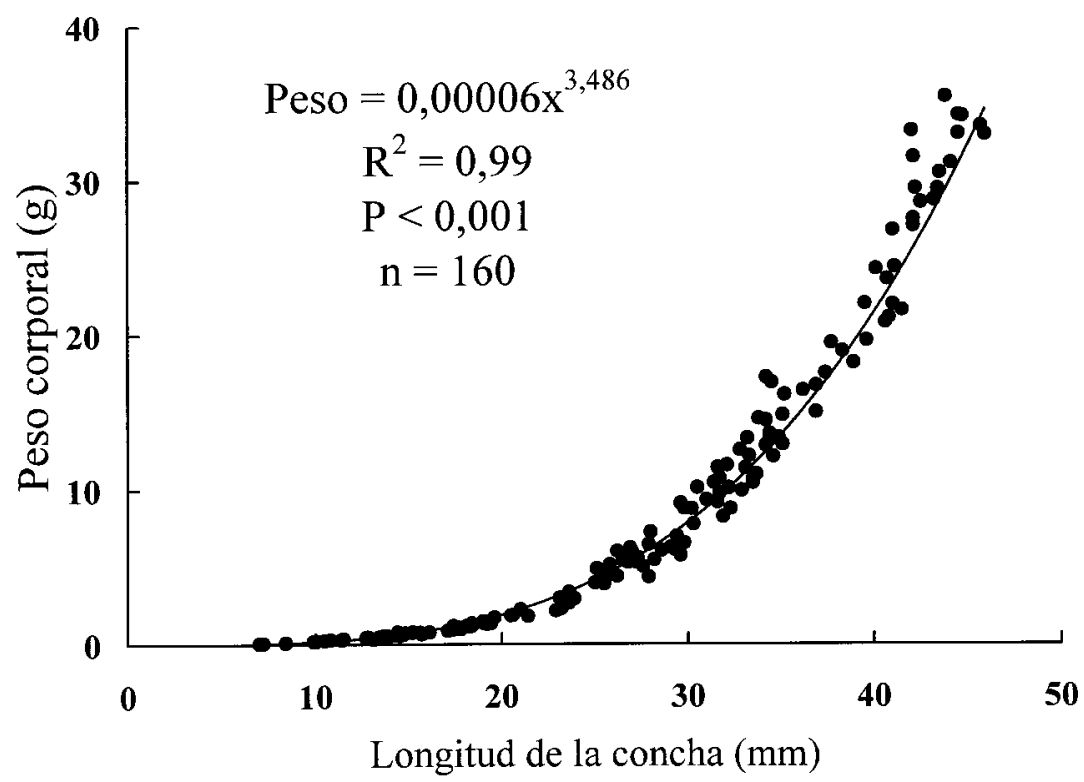

Fig. 4: Relación entre el largo de la concha y el peso húmedo de Acanthina monodon recolectados en el intermareal rocoso de Chile central.

Relationship between shell length and wet weight of Acanthina monodon collected from intertidal rocky shores of central Chile.

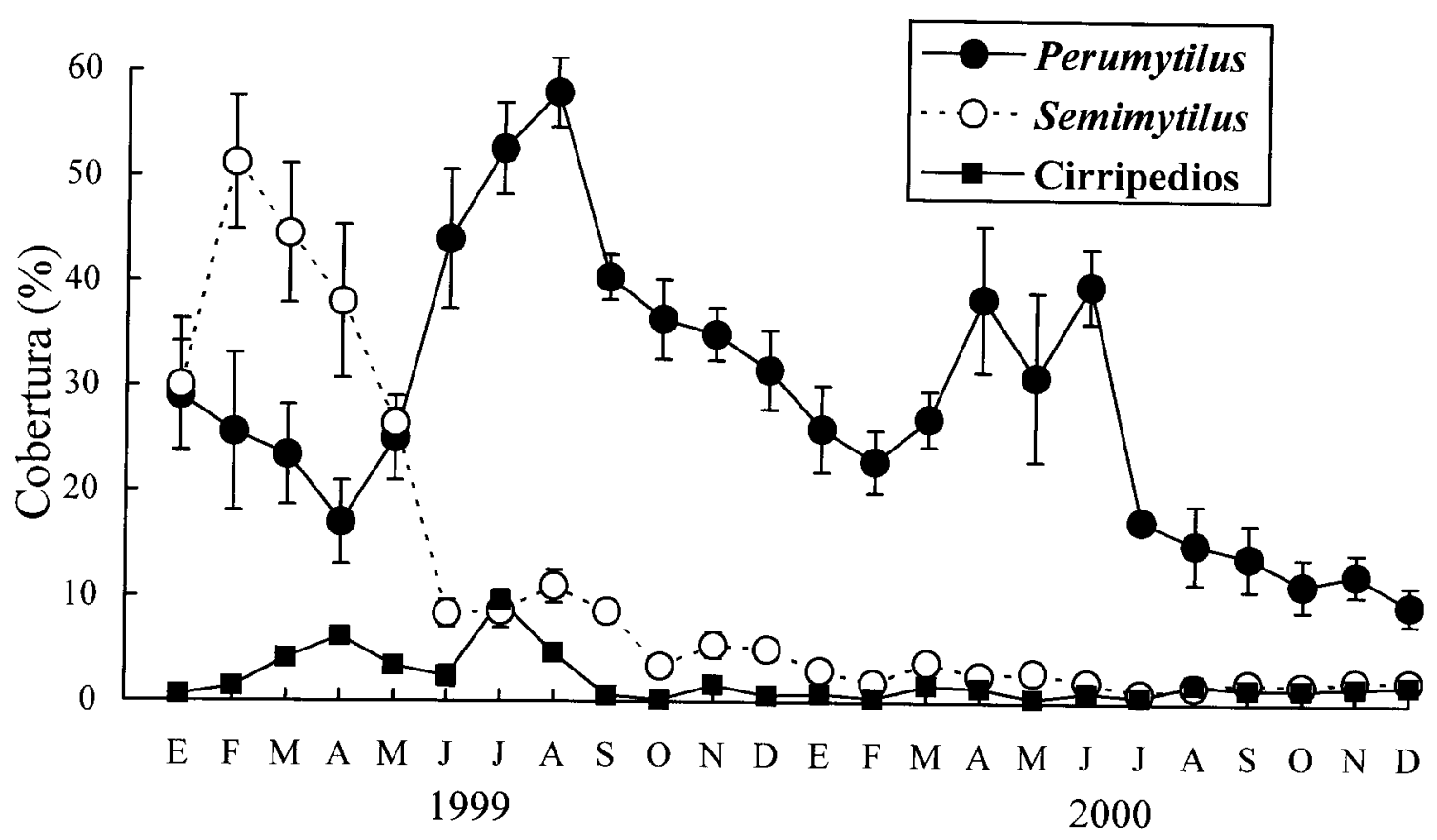

Fig. 5: Cobertura porcentual de presas durante 1999 y 2000 en el intermareal rocoso de Chile central. Las barras indican \pm 1 error estándar.

Percent cover of prey during 1999 and 2000 on intertidal rocky shores of central Chile. Bars indicate \pm 1 standard error. 

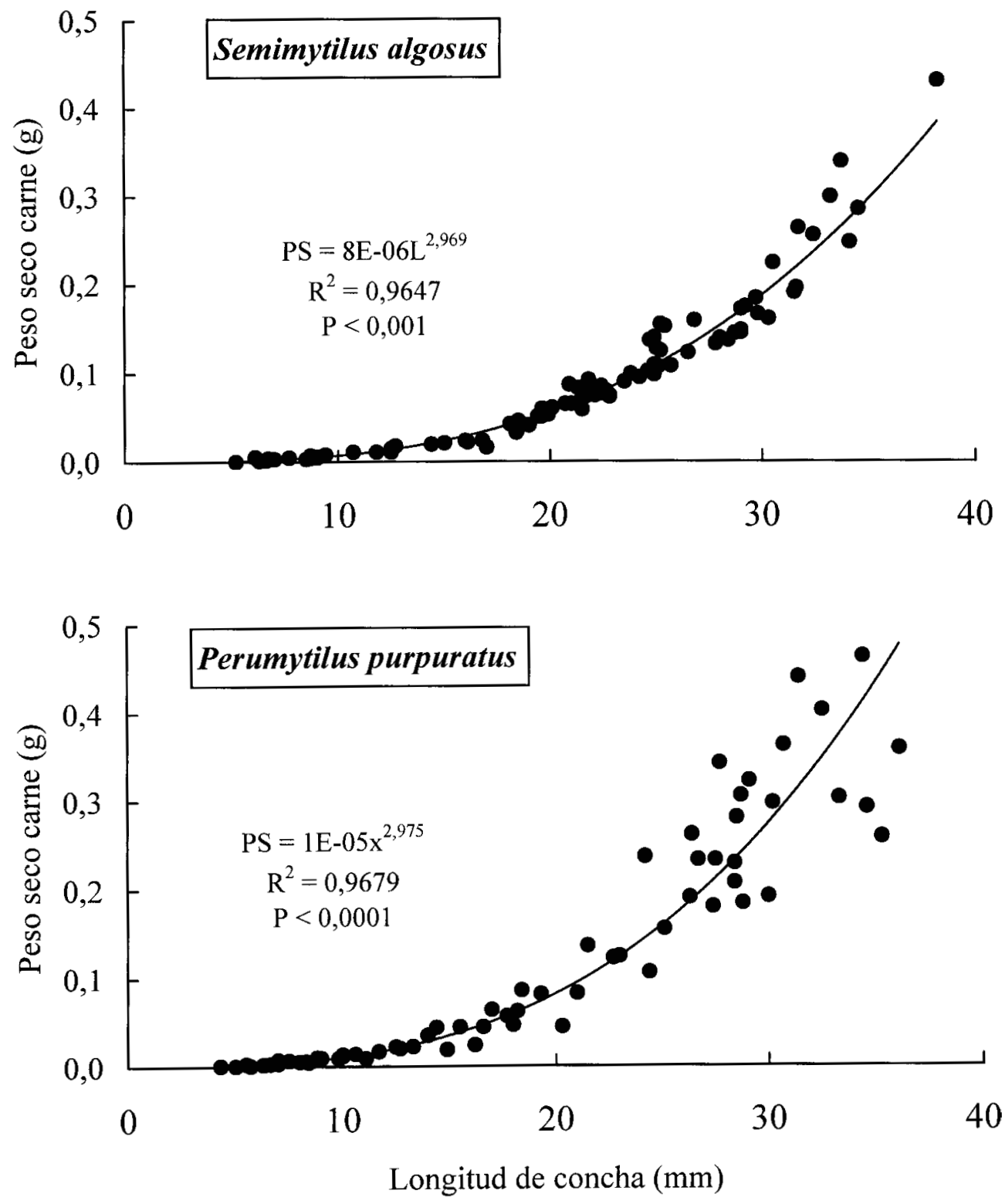

Fig. 6: Relación entre el tamaño corporal y el peso seco de los tejidos blandos para dos especies de mitílidos presentes en el intermareal rocoso de Chile central.

Relationship between body size and dry weight of soft tissues for two mussel species from the intertidal rocky shores of central Chile.

Grosor de la concha

Las relaciones entre la longitud y el grosor de la concha presentaron diferencias significativas entre ambas especies de mitílidos (ANCOVA: $\left.\mathrm{F}_{1,57}=140,6 ; \mathrm{P}<0,0001\right)$. La concha de los individuos de la especie $P$. purpuratus presentó valores de grosor mayores que los observados para S. algosus (Fig. 7).
Tasa de consumo de A. monodon

La tasa de consumo de A. monodon en condiciones de laboratorio presentó diferencias significativas entre individuos juveniles $\mathrm{y}$ adultos (ANDEVA, $\mathrm{F}_{1,44}=25,20 ; \mathrm{P}<0,001$ ). Los individuos juveniles, con masa corporal menor a $10 \mathrm{~g}$, mostraron una tasa de consumo promedio de $7,44 \mathrm{mg}$ día $^{-1}(\mathrm{DE}=3,06)$. Los 


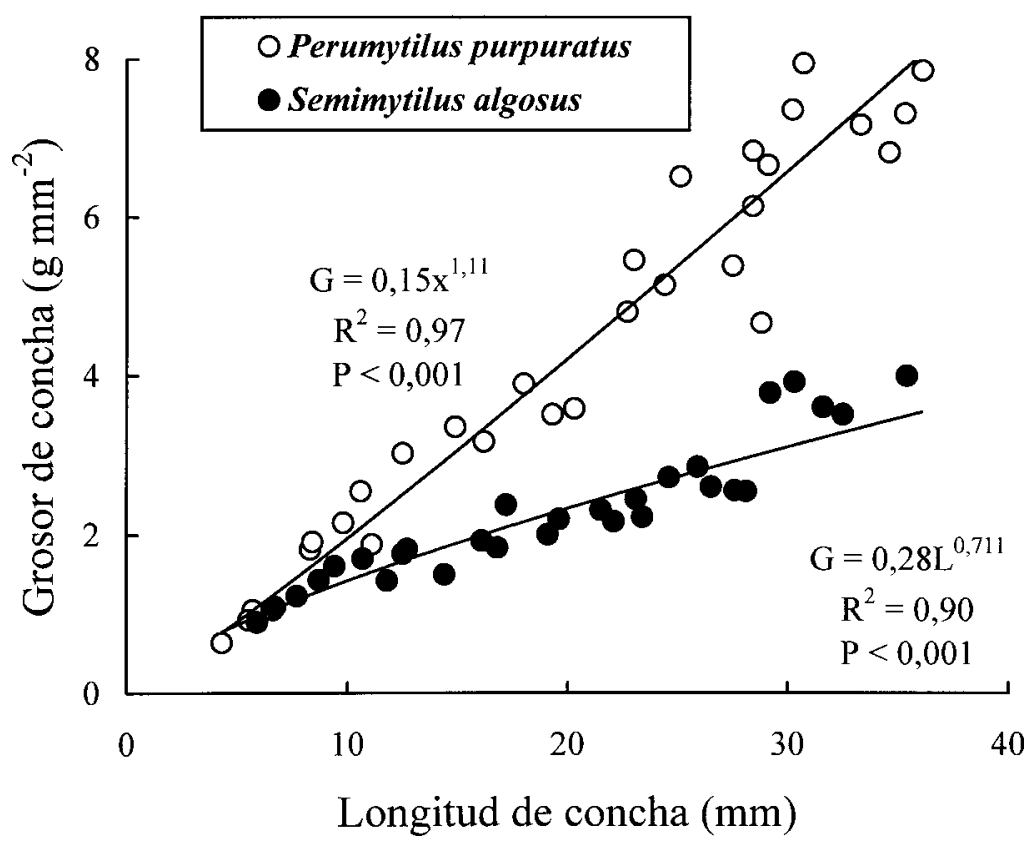

Fig. 7: Relación entre el tamaño y el grosor de la concha para dos especies de mitílidos del intermareal rocoso de Chile central.

Relationship between size and thickness of shells for two mussel species of the intertidal rocky shores of central Chile.

individuos adultos, con masa corporal mayor a $10 \mathrm{~g}$, presentaron una tasa de consumo promedio de 19,32 $\mathrm{mg} \mathrm{día}^{-1}(\mathrm{DE}=4,367$; Fig. 8). Las relaciones entre el tamaño corporal y la tasa de consumo obtenidas con los mitílidos $S$. algosus y $P$. purpuratus no se diferenciaron significativamente (ANCOVA, $\mathrm{F}_{1,37}=1,16 ; \mathrm{P}=$ 0,286; Fig. 8).

\section{Preferencias alimentarias}

Los individuos juveniles presentaron una preferencia significativa por el mitílido $S$. algosus, mientras que entre las otras dos presas disponibles $P$. purpuratus y $B$. granulata no se detectaron preferencias significativas (prueba de Friedman $=13,4 ; \mathrm{gl}=2 ; \mathrm{P}=0,001$; Fig. 9). Del mismo modo, los individuos adultos presentaron una preferencia significativa por $S$. algosus en comparación a los otros dos tipos de presas, entre las cuales no fue posible discriminar algún tipo de preferencia estadísticamente significativa (prueba de Friedman $=10,05 ; \mathrm{gl}=2 ; \mathrm{P}=0,006$; Fig. 9).

\section{Preferencias por tamaños de presa}

Los individuos adultos de A. monodon mostraron una preferencia significativa por las presas de tamaño corporal entre 20 y $25 \mathrm{~mm}$ de longitud. No fue posible discriminar preferencias significativas entre presas de tamaños pequeñas y grandes (Fig. 10). Esta preferencia significativa por las presas de tamaño medianas se observó indistintamente tanto para los individuos que estaban consumiendo $S$. algosus (prueba de Friedman = 9,65; $\mathrm{gl}=2 ; \mathrm{P}=0,008 ;$ Fig. 10) como los que depredaban sobre $P$. purpuratus (prueba de Friedman $=8,45 ; \mathrm{gl}=2 ; \mathrm{P}=0,015 ;$ Fig. 10).

\section{Tiempo de ingestión y manipulación de presas}

En los individuos adultos de A. monodon la relación entre el tiempo de ingestión y el tamaño de presa muestra que el tiempo usado en manipular e ingerir la presa presenta un aumento en relación directa con el tamaño corporal de la presa consumida. Esta relación fue independiente del tipo de presa evaluado (Fig. 11). Sin embargo, el tiempo usado en manipular e ingerir una presa de la especie $S$. algosus fue menor que el tiempo empleado en consumir una de $P$. purpuratus del mismo tamaño corporal (ANCOVA, $\mathrm{F}_{1,30}=5,40 ; \mathrm{P}=$ $0,027)$. Por otra parte, al incorporar en el análisis de manipulación el aporte energético de las presas se observó que las mayores 


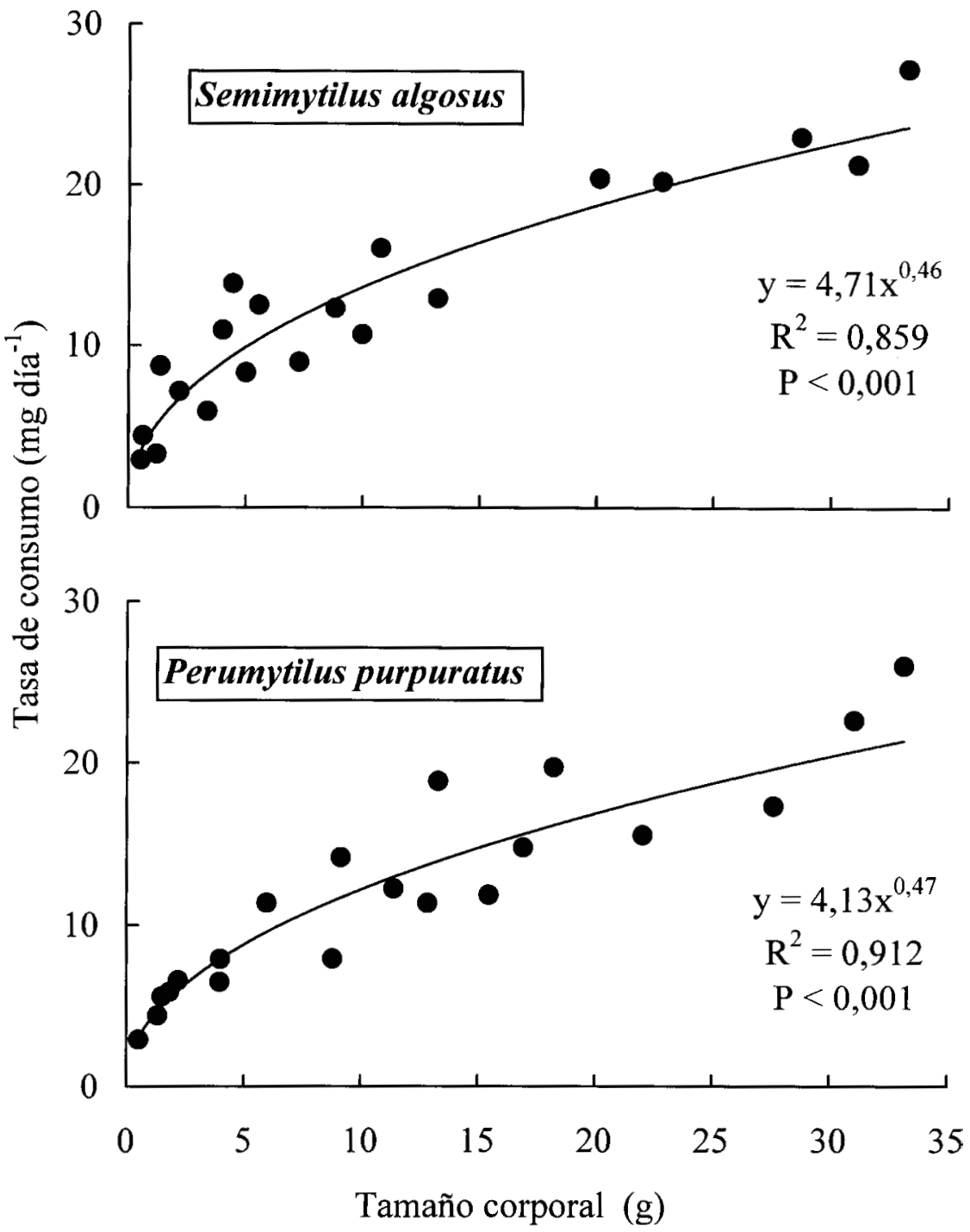

Fig. 8: Relación entre el tamaño corporal de Acanthina monodon y la tasa de consumo de alimento, evaluada con dos especies de mitílidos.

Relationship between the body size of Acanthina monodon and food consumption rate, evaluated with two mussel species.

rentabilidades (ganancia energética dividido por el tiempo de manipulación) fueron obtenidas a partir de presas de entre 20 a 25 $\mathrm{mm}$ de longitud de concha. Esta relación fue observada indistintamente tanto para $S$. algosus como para $P$. purpuratus. Sin embargo, la relación derivada entre la rentabilidad y el tamaño corporal de la presa con $S$. algosus fue significativamente distinta de la relación obtenida con $P$. purpuratus (ANCOVA, $\mathrm{F}_{1,30}=$ 5,02; $\mathrm{P}=0,032)$. De este modo, las rentabilidades energéticas obtenidas cuando la presa evaluada fue $S$. algosus resultaron mayores a las obtenidas con $P$. purpuratus.

\section{DISCUSIÓN}

Los valores de densidad promedio obtenidos para las poblaciones de A. monodon que habitan en las plataformas rocosas intermareales de Chile central concuerdan con los valores registrados para esta variable en los muestreos realizados en sitios ubicados en los alrededores de la zona de Valdivia en el sur de Chile (Dye 1991, López et al. 1995, Moreno 1995). La distribución espacial que ocupa $A$. monodon en dichas plataformas en el sur de Chile es mucho más amplia que la distribución observada para la zona central, ya que para 

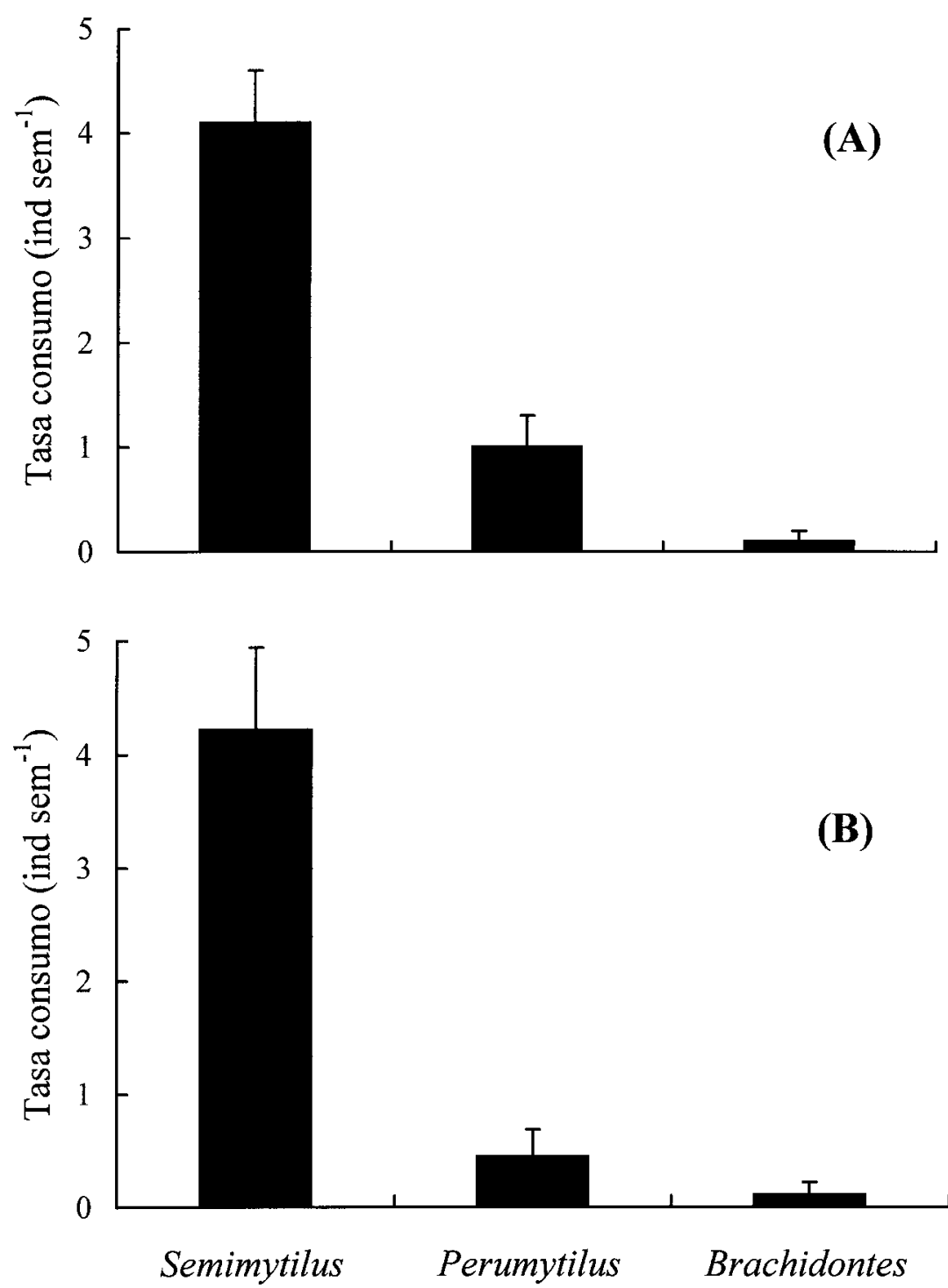

Fig. 9: Preferencias alimentarias de individuos juveniles (A) y adultos (B) de Acanthina monodon. Las líneas sobre las barras indican un error estándar.

Feeding preference of juvenile (A) and adult (B) of Acanthina monodon. The lines above bars indicate one standard error.

muestreos realizados en la Bahía de Metri en el Seno Reloncaví (sur de Chile), este gastrópodo se distribuía en el nivel medio del intermareal alcanzando las mayores densidades entre los 1 y $4 \mathrm{~m}$ sobre la cota de marea cero (López et al. 1995). El hecho que A. monodon ocupe una franja más restringida en el intermareal de Chile central podría estar indicando el efecto de regímenes ambientales diferentes, entre los que podría tener un importante efecto la temperatura y humedad ambiental, ya que para otros gastrópodos este factor limita la distribución espacial hacia niveles superiores del intermareal (Little \& Kitching 1996, Barnes \& Hughes 1999). Esta hipótesis podría ser respaldada por el hecho de que durante la estación de invierno fue cuando A. monodon alcanzó la mayor altura en las plataformas intermareales en Chile central, lo que concuerda con menores valores de temperatura tanto ambiental como de la superficie de las rocas (Soto \& Bozinovic 1998).

El gastrópodo A. monodon es un depredador que ejerce su acción exclusivamente sobre invertebrados sésiles que ocupan espacio primario en las plataformas intermareales de 

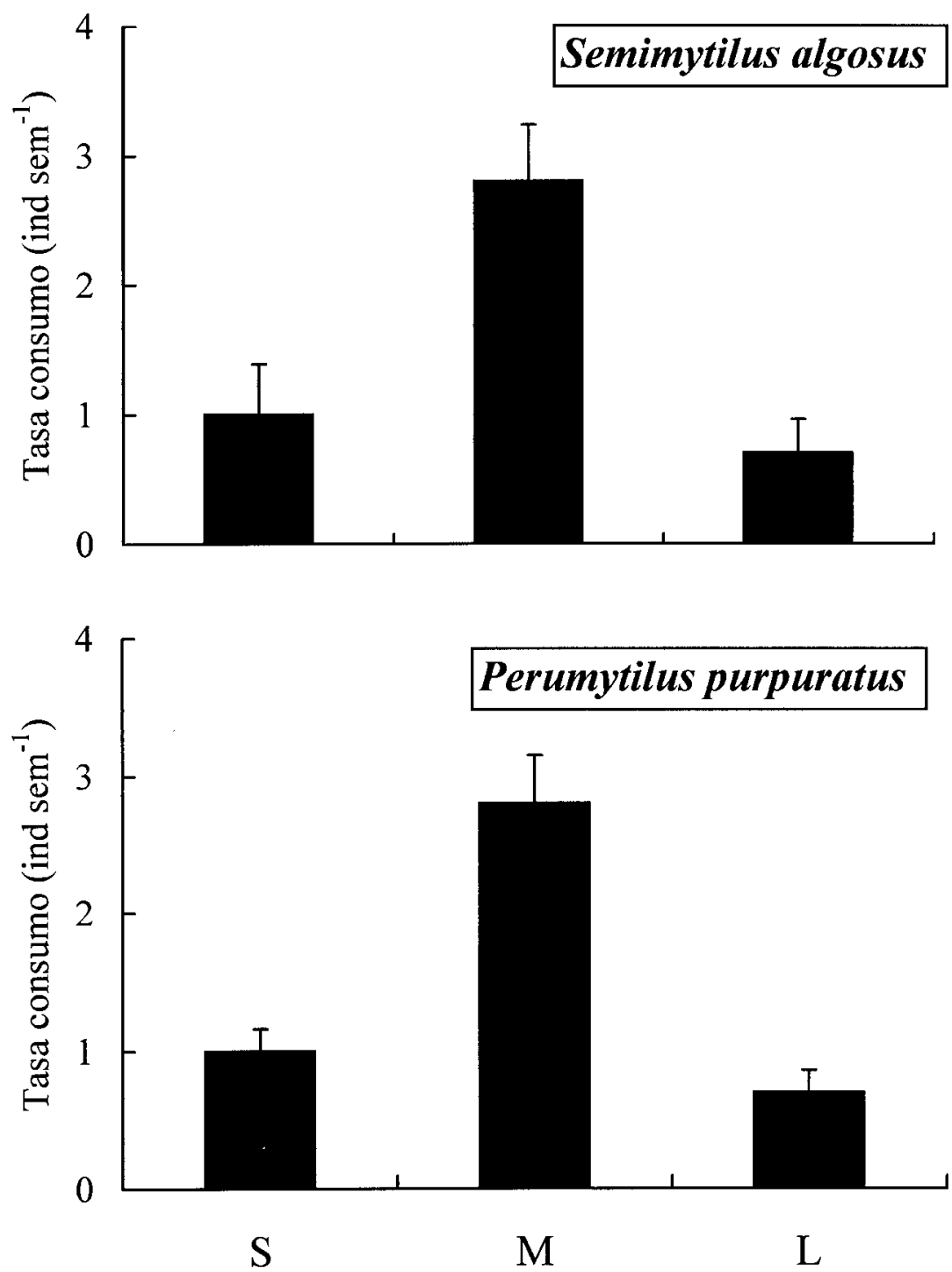

Fig. 10: Preferencias por tamaños de presas en individuos adultos de Acanthina monodon. Donde S = presas pequeñas $(15-20 \mathrm{~mm}), \mathrm{M}=$ presas medianas $(20-25 \mathrm{~mm}), \mathrm{y} \mathrm{L}=$ presas grandes $(25-30$ $\mathrm{mm})$. Las líneas sobre las barras indican un error estándar.

Prey size preference for adult of Acanthina monodon. Where $\mathrm{S}=$ small preys $(15-20 \mathrm{~mm}), \mathrm{M}=$ medium preys $(20-25 \mathrm{~mm})$, and $\mathrm{L}=$ large preys $(25-30 \mathrm{~mm})$. Lines above the bars indicate one standard error.

Chile central. Su dieta está compuesta de cirripedios como de mitílidos. Los mitílidos constituyen aproximadamente el $95 \%$ de la dieta de este caracol, lo cual concuerda con los datos entregados por Moreno (1995) quien señaló que un $87 \%$ de las presas consumidas por individuos de $A$. monodon corresponden a mitílidos mientras que solo un $11,6 \%$ correspondían a cirripedios. Aunque en terreno el alto consumo de mitílidos es un patrón temporalmente consistente para A. monodon, al realizar un análisis caracterizando las especies consumidas a lo largo del tiempo evaluado, se observó que existían variaciones en las especies de mitílidos que formaban parte de su dieta. Las variaciones obtenidas pueden estar asociadas a los cambios que experimenta la oferta de las presas a lo largo del tiempo en el sitio de muestreo (Fig. 5). Así, cuando existió una mayor cobertura de $S$. algosus (verano y otoño de 1999) esta fue la especie de mitílido mayoritariamente consumida por Acanthina en terreno. Del mismo modo, cuando existió una mayor cobertura de $P$. purpuratus esta fue la 


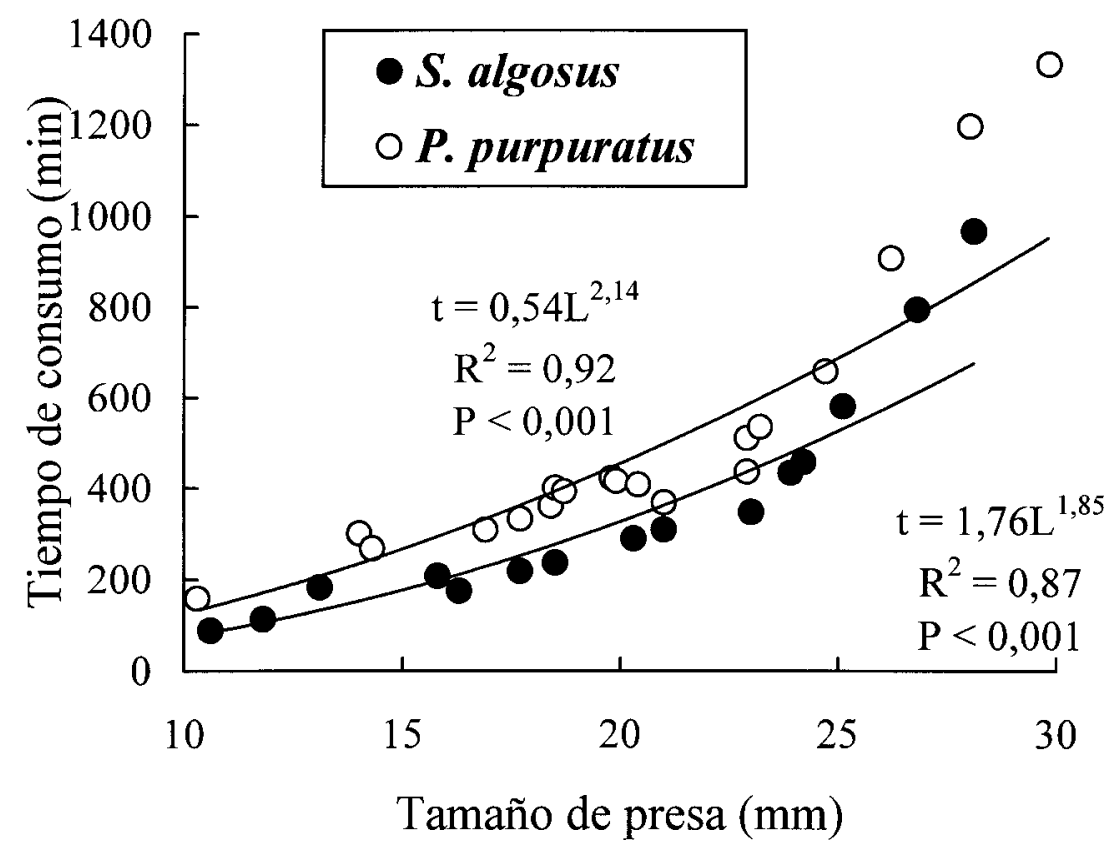

Fig. 11: Tiempo empleado por individuos adultos de Acanthina monodon en el consumo de dos especies de mitílidos.

Time spent by adult Acanthina monodon feeding on two mussel species.

presa sobre la cual se ejerció la mayor frecuencia de consumo. Esta evidencia podría indicar una expresión particular de una respuesta de tipo funcional por parte de $A$. monodon, ya que la oferta de presas modificaría la conducta del depredador ("switching behavior"; Murdoch 1969, Hughes \& Croy 1993). En consecuencia, los cambios en la selección de presas dependerían de la frecuencia relativa de los distintos tipos de mitílidos presentes en el terreno.

Un aspecto importante de las observaciones de la frecuencia estacional de consumo de presas de A. monodon, está dado por la alta variabilidad temporal que puede experimentar la ingestión de determinados tipos de presas en terreno. En general, la mayoría de las investigaciones cuantitativas acerca de la conducta trófica de una determinada especie animal, independientemente del ambiente en el cual habiten, están basadas en evaluaciones puntuales que solo representan la ventana temporal en la cual fueron realizadas (véase Jaksic et al. 1997 para una excepción). Entonces, cualquier tipo de extrapolación o predicción de patrones hacia escalas mayores que aquellas a las cuales fueron realizadas, deberían ser consideradas cuidadosamente (Underwood \& Petraitis 1993).
Acanthina monodon realiza sus actividades de forrajeo (búsqueda, manipulación e ingestión de presas) durante períodos nocturnos. Sin embargo, si la presa seleccionada es de un tamaño corporal grande, los caracoles permanecen ingiriendo durante el día. Este patrón conductual nocturno concuerda con lo observado en otros gastrópodos intermareales de la costa de Chile, entre los cuales se pueden contar Concholepas concholepas y Crassilabrum crassilabrum (Castilla et al. 1979, R.E. Soto observación personal). En el caso del gastrópodo murícido C. concholepas, los estudios en el laboratorio demuestran que las actividades de alimentación, copulación y locomoción ocurren preferentemente durante períodos de oscuridad e independiente de la hora solar (Castilla \& Guisado 1979, Castilla et al. 1979). Sin embargo, el ritmo de alimentación observado en A. monodon se contrapone al patrón descrito para otras especies del genero Acanthina del hemisferio Norte, como ocurre con Acanthina (Nucella) lapillus que realiza sus actividades de forrajeo durante períodos de inmersión o marea alta (Burrow \& Hughes 1991).

La relación entre la longitud y el peso corporal para los individuos de A. monodon provenientes de Chile central se diferencia 
significativamente de la ecuación publicada por Poblete et al. (1987) para caracoles de la misma especie del sur de Chile. Las diferencias podrían ser debidas a que estos autores en su investigación consideraron solo individuos desde los $18 \mathrm{~mm}$ de longitud de la concha; además en el estudio incorporaron algunos individuos que presentaban tamaños superiores a los $50 \mathrm{~mm}$. Esto se traduce en que la curva propuesta por Poblete et al. (1987) tiende a sobreestimar el peso corporal de los individuos más pequeños. Es importante destacar que algunos registros realizados para esta relación con individuos de otras localidades de Chile central, no se diferenciaban significativamente de los parámetros obtenidos con los individuos de Las Cruces (Soto 2001).

Bajo condiciones de laboratorio, la tasa de ingestión de los individuos adultos de $A$. monodon presentó valores cercanos a los $425 \mathrm{~J}$ día $^{-1}$. Las tasas de consumo en murícidos han sido medidas en diversos estudios experimentales previos. Menge (1978) estudió la tasa de consumo de $N$. lapillus alimentados con $M$. edulis mediante cajas de exclusión instaladas en el intermareal rocoso. Luego de realizar las conversiones a unidades comparables sus estimaciones alcanzaron a valores entre 0 y $60 \mathrm{~J}$ día ${ }^{-1}$. En evaluaciones realizadas en el laboratorio por Bayne \& Scullard (1978) demostraron tasas de consumo para Thais lapillus de 60, 80, y $120 \mathrm{~J} \mathrm{día}^{-1}$ a 9,15 y $20{ }^{\circ} \mathrm{C}$, respectivamente. Los valores de tasa de consumo obtenidos en este trabajo podrían ser considerados como elevados en comparación a los otros murícidos evaluados. Sin embargo, estas variaciones pueden relacionarse con diferencias en el tamaño corporal de los individuos adultos de las distintas especies de murícidos estudiados (Vermeij 1978).

La relación entre la tasa de consumo y el peso corporal para $A$. monodon presentó un exponente con un valor aproximado de 0,47 independientemente del tipo de mitílido utilizado como presa (Fig. 8). En otros estudios realizados con gastrópodos marinos carnívoros los exponentes que relacionan la tasa de ingestión y el tamaño corporal ( $g$ ) han alcanzado valores de entre 0,40 a 0,56 para $N$. lapillus forrajeando sobre M. edulis (Bayne \& Scullard 1978). En el caso del murícido $C$. concholepas que se alimenta de $M$. chilensis, Navarro \& Torrijos (1995) obtuvieron valores de exponentes que alcanzaron a 0,$52 ; 0,54$; 0,61 y 0,55 para las estaciones de primavera, verano, otoño e invierno respectivamente.

Los resultados obtenidos en los experimentos de preferencias alimentarias demuestran que $A$. monodon selecciona las presas que consume tanto por especie como por tamaños. Los experimentos de selección de presas para este gastrópodo determinaron que presenta una preferencia significativa por el mitílido $S$. algosus, lo cual concuerda con los registros de preferencias realizados con otros murícidos que habitan en la costa de Chile. Por ejemplo, Méndez \& Cancino (1990) demostraron, mediante experimentos en laboratorio, que los individuos juveniles y postmetamórficos de $C$. concholepas seleccionaban preferentemente a la presa $S$. algosus. Del mismo modo, Gutiérrez \& Gallardo (1999) han informado que los individuos juveniles del gastrópodo murícido Chorus giganteus también presentan una preferencia significativa por el mitílido S. algosus. La marcada preferencia que presentan estas especies de gastrópodos por $S$. algosus estaría asociada al menor tiempo empleado en la apertura e ingestión de esta presa, principalmente debido al menor grosor que presenta su concha en comparación a otras especies de mitílidos que han sido presentadas como presas alternativas (Fig. 10). De una manera similar, el murícido Acanthina spirata presenta una fuerte y marcada preferencia por el mitílido $M$. edulis en comparación con $M$. californianus, el cual presenta una concha más gruesa, que resulta más difícil de perforar y consumir (Murdoch 1969, Hughes 1986).

Al analizar las preferencias alimentarias de A. monodon se puede indicar que este gastrópodo presenta una conducta de forrajeo que maximiza el retorno de energía mediante la selección de la especie y los tamaños de presas con las cuales obtiene una mayor rentabilidad energética. Sin embargo, es importante considerar que las evaluaciones realizadas en el presente trabajo fueron efectuadas bajo condiciones de laboratorio. Por esto, futuras investigaciones relacionadas con la conducta de forrajeo de este gastrópodo deberían abordar aspectos relacionados con el efecto de las restricciones impuestas tanto por factores físicos como biológicos sobre las respuestas conductuales asociadas a la búsqueda e ingestión de alimento. Por ejemplo, es interesante notar cómo A. monodon varía temporalmente el consumo de las distintas especies presa según la abundancia relativa de estas, lo cual implicaría ajustar su conducta de forrajeo de acuerdo a la disponibilidad de los recursos alimentarios.

\section{AGRADECIMIENTOS}

Se agradecen los valiosos comentarios realizados por dos revisores anónimos, los 
cuales mejoraron considerablemente el contenido del manuscrito. Este trabajo fue iniciado gracias a los aportes del proyecto FONDECYT 2980020 y fue finalizado y escrito gracias al proyecto FONDECYT 3010045.

\section{LITERATURA CITADA}

BAYNE BL \& C SCULLARD (1978) Rates of feeding by Thais (Nucella) lapillus (L.). Journal of Experimental Marine Biology and Ecology 32: 113-129.

BARNES RSK \& RN HUGHES (1999) An introduction to marine ecology. Third edition. Blackwell Science, Oxford, United Kingdom. 286 pp.

BURROWS MT \& RN HUGHES (1991) Optimal foraging decisions by dogwhelks, Nucella lapillus (L.): influences of mortality risk and rate-constrained digestion. Functional Ecology 5: 461-475.

CARRIKER MR (1981) Shell penetration and feeding by naticacean and muricacean predatory gastropods: a synthesis. Malacologia 20: 403-422.

CÁCERES CW, LS FUENTES \& FP OJEDA (1994) Optimal feeding strategy of the temperate herbivorous fish Aplodactylus punctatus: the effects of food availability on digestive and reproductive patterns. Oecologia 99: 118-123.

CASTILLA JC (1981) Perspectiva de investigación en estructura y dinámica de comunidades intermareales rocosas de Chile central. II. Depredadores de alto nivel trófico. Medio Ambiente (Chile) 5: 190-215.

CASTILLA JC (1988) Earthquake-caused coastal uplift and its effects on rocky intertidal kelp communities. Science 242: 440-443.

CASTILLA JC (1999) Coastal marine communities: trends and perspectives from human-exclusion experiments. Trends in Ecology and Evolution 14: 280-283.

CASTILlA JC \& C GUISADO (1979) Conducta de alimentación nocturna de Concholepas concholepas (Mollusca: Gastropoda: Muricidae). Biología Pesquera (Chile) 12: 125-130.

CASTILLA JC, C GUISADO \& J CANCINO (1979) Aspectos ecológicos y conductuales relacionados con la alimentación de Concholepas concholepas (Mollusca: Gastropoda: Muricidae). Biología Pesquera (Chile) 12: 99-114.

CASTILLA JC \& RT PAINE (1987) Predation and community organization on Eastern Pacific, temperate zone, rocky intertidal shores. Revista Chilena de Historia Natural 60: 131-151.

CONOVER MR (1981) Practical nonparametric statistics. John Wiley \& Sons, New York, New York, USA. 493 pp.

DURÁN LR \& JC CASTILLA (1989) Variation and persistence of the middle rocky intertidal communities of central Chile with and without human harvesting. Marine Biology 103: 555-562.

DYE AH (1991) Food preferences of Nucella crassilabrum and juveniles of Concholepas concholepas (Gastropoda: Muricidae) from a rocky shore in southern Chile. Journal of Molluscan Studies 57: 301-307.

ELNER RW \& RN HUGHES (1978) Energy maximization in the diet of the shore crab, Carcinus maenas. Journal of Animal Ecology 47: 103-116.

FAIRWEATHER PG (1985) Differential predation on alternative prey, and the survival of rocky intertidal organisms in NSW. Journal of Experimental Marine Biology and Ecology 89: 145-156.
FOSTER MS, C HARROLD \& DD HARDIN (1991) Point vs. quadrant estimates of the cover of sessile marine organisms. Journal of Experimental Marine Biology and Ecology 146: 193-203.

GUIÑEZ RE (1996) Dinámica poblacional del chorito maico, Perumytilus purpuratus (Lamarck 1819) (Bivalvia: Mytilidae), en gradientes de exposición al oleaje. Tesis de doctorado, Pontificia Universidad Católica de Chile, Santiago, Chile. 213 pp.

GUTIÉRREZ RM \& CS GALLARDO (1999) Prey attack, food preference and growth in juveniles of the edible muricid snail, Chorus giganteus. Aquaculture 174: 69-79.

HUGHES RN (1986) A functional biology of marine gastropods. The Johns Hopkins University Press, Baltimore, Maryland, USA. $245 \mathrm{pp}$.

HUGHES RN (1988) Optimal foraging in the intertidal environment: evidence and constraints. In: Chelazzi $\mathrm{G} \& \mathrm{M}$ Vannini (eds) Behavioural adaptation to intertidal life: 265-282. Plenum Press, New York, New York, USA.

HUGHES RN (1993) Diet selection: an interdisciplinary approach to foraging behaviour. Blackwell Scientific Publications, Oxford, United Kingdom. $221 \mathrm{pp}$.

HUGHES RN \& MI CROY (1993) An experimental analysis of frequency dependent predation (switching) in the 15-spined stickleback, Spinachia spinachia. Journal of Animal Ecology 62: 341-352.

HUGHES RN \& SB DUNKIN (1984) Behavioural components of prey selection by dogwhelks, Nucella lapillus (L.), feeding on mussels, Mytilus edulis (L.), in the laboratory. Journal of Experimental Marine Biology and Ecology 79: 45-68.

JAKSIC FM, SI SILVA, PL MESERVE \& JR GUTIÉRREZ (1997) A long-term study of vertebrate predator response to an El Niño (ENSO) disturbance in western South America. Oikos 78: 341-354.

KISLALIOGLU M \& RN GIBSON (1975) Prey 'handling time' and its importance in food selection by the 15-spined stickleback Spinachia spinachia (L.). Journal of Experimental Marine Biology and Ecology 25: 159-169.

KERSTEIN M \& W VISSER (1996) The rate of food processing in the oystercatcher: food intake and energy expenditure constrains by a digestive bottleneck. Functional Ecology 10: 440-448.

KOHN AJ (1983) Feeding biology of gastropods. The Mollusca 5: 1-63.

KREBS JR \& NB DAVIES (1997) Behavioural ecology: an evolutionary approach. Fourth edition. Blackwell Science, Oxford, United Kingdom. 456 pp.

LEMIRE M \& JH HIMMELMAN (1996) Relation of food preference to fitness for the green sea urchin, Strongylocentrotus droebachiensis. Marine Biology 127: 73-78.

LITTLE C \& JA KITCHING (1996) The biology of rocky shores. Oxford University Press, Oxford, United Kingdom. 240 pp.

LÓPEZ DA, ML GONZÁLEZ, MV VIAL \& RW SIMPFERDORFER (1995) Sublethal effects provoked by the presence of the predator Nucella crassilabrum (Lamarck) upon the mussel Perumytilus purpuratus (Lamarck) in Chile. Revista Chilena de Historia Natural 68: 469-475.

MÉNDEZ MA \& JM CANCINO (1990) Preferencias alimentarias de ejemplares postmetamórficos y juveniles de Concholepas concholepas. Revista de Biología Marina (Chile) 25: 109-120.

MENGE BA (1978) Predation intensity in a rocky intertidal community. Effect of an algal canopy, 
wave action and desiccation on predator feeding rates. Oecologia 34: 17-35.

MORENO CA (1995) Macroalgae as a refuge from predation for recruits of the mussel Choromytilus chorus (Molina, 1782) in Southern Chile. Journal of Experimental Marine Biology and Ecology 191: 181-193.

MURDOCH WW (1969) Switching in general predators: experiments on predator specificity and stability of prey populations. Ecological Monographs 39: 335-354

NAVARRO JM \& RA TORRIJOS (1995) Fisiología energética de Concholepas concholepas (Bruguiere, 1789) (Gastropoda: Muricidae) en la bahía de Yaldad, sur de Chile. Revista Chilena de Historia Natural 68: 61-77.

PALMER AR (1984) Prey selection by thaidid gastropods: some observational and experimental field test of foraging models. Oecologia 62: 162-172.

PALMER AR (1988) Feeding biology of Ocenebra lurida (Prosobranchia: muricacea): diet, predator-prey size relations, and attack behavior. The Veliger 31: 192-203.

POBLETE T, H TOLEDO, R ARTEAGA, R CÁRDENAS \& M TOLEDO (1987) Estimación de la estructura por clases anuales de tamaño en una población de Nucella crassilabrum (Gastropoda, Muricidae). Biota (Chile) 3: 9-31.

ROA R (1992) Design and analysis of multiple-choice feeding preferences experiments. Oecologia 89: 509-515.

ROCHETTE R \& LM DILL (2000) Mortality, behavior and the effects of predators on the intertidal distribution of littorinid gastropods. Journal of Experimental Marine Biology and Ecology 253: 165-191.

SANTELICES B (1991) Litoral and sublittoral communities of continental Chile. In: Mathieson AC \& PH Nienhuis (eds) Intertidal and littoral ecosystems: 347-369. Elsevier, Amsterdam, The Netherlands.

SERVICIO HIDROGRÁFICO Y OCEANOGRÁFICO DE LA ARMADA DE CHILE (1999) Tablas de marea de la costa de Chile, Publicación 3009, Valparaíso, Chile. 247 pp.

SCHOENER TW (1971) Theory of feeding strategies. Annual Review of Ecology and Systematics 2: 369-404

SEED R \& RN HUGHES (1995) Criteria for prey sizeselection in molluscivorous crabs with contrasting claw morphologies. Journal of Experimental Marine Biology and Ecology 193: 177-195.
SERRA G, G CHELAZZI \& JC CASTILLA (1997) Effects of experience and risk of predation on the foraging behaviour of the South-eastern Pacific muricid Concholepas concholepas (Mollusca: Gastropoda). Journal of Animal Ecology 66: 876-884.

SOTO RE (2001) Rol de los componentes asociados a la experiencia ingestiva y el riesgo de mortalidad sobre la conducta y decisiones de forrajeo del gastrópodo murícido Nucella crassilabrum en el intermareal rocoso de Chile central. Tesis de doctorado, Pontificia Universidad Católica de Chile, Santiago, Chile. 247 pp.

SOTO RE \& F BOZINOVIC (1998) Behavioral thermoregulation of the periwinkle Nodilittorina peruviana inhabiting the rocky intertidal of central Chile: a laboratory and field study. Revista Chilena de Historia Natural 71: 375-382.

STEPHENS DW \& JR KREBS (1986) Foraging theory. Princeton University Press, Princeton, New Jersey, USA. $247 \mathrm{pp}$.

SUTHERLAND WJ (1982) Do oystercatchers select the most profitable cockles? Animal Behaviour 30: 857-861.

UNDERWOOD AJ (1997) Experiments in ecology: their logical design and interpretation using analysis of varianza. Cambridge University Press, Cambridge, United Kingdom. 504 pp.

UNDERWOOD AJ \& PS PETRAITIS (1993) Structure of intertidal assemblages in different locations: how can local processes be compared? In: Ricklefs R \& D Schluter (eds) Species diversity in ecological communities: 39-51. University Chicago Press, Chicago, Illinois, USA.

VADAS RL (1977) Preferential feeding: an optimization strategy in sea urchins. Ecological Monographs 47: 337-371.

VERMEIJ GJ (1978) Biogeography and adaptation: patterns of marine life. Harvard University Press, Cambridge, United Kingdom. 332 pp.

WEST L (1986) Interindividual variation in prey selection by the snail Nucella (=Thais) emarginata. Ecology 67: 798-809.

WIETERS EA \& SA NAVARRETE (1998) Spatial variability in prey preferences of the intertidal whelks Nucella caniculata and Nucella emarginata. Journal of Experimental Marine Biology and Ecology 222: 133-148.

ZAR JH (1996) Biostatistical analysis. Third edition. Prentice-Hall International, Englewood Cliffs, New Jersey, USA. 662 pp. 